\title{
Research on Fault Diagnosis of Flexible Material R2R Manufacturing System Based on Quality Control Chart and SoV
}

\author{
Yaohua Deng $\left(\mathbb{1},{ }^{1,2}\right.$ Na Zhou $\left(\mathbb{1},{ }^{1}\right.$ Xiali Liu $(\mathbb{1}){ }^{3}$ and Qiwen Lu $\mathbb{D}^{3}$ \\ ${ }^{1}$ School of Electro-Mechanical Engineering, Guangdong University of Technology, Guangzhou, Guangdong, China \\ ${ }^{2}$ Department of Mechanical Engineering, University of Michigan, Ann Arbor, MI, USA \\ ${ }^{3}$ Foshan World Intelligent Technology Co., Ltd., Foshan, Foshan 528000, China
}

Correspondence should be addressed to Na Zhou; zna0202@163.com, Xiali Liu; xialil@fswitc.com, and Qiwen Lu; 24084480@qq.com

Received 21 February 2018; Accepted 3 April 2018; Published 21 May 2018

Academic Editor: Guangming Xie

Copyright (c) 2018 Yaohua Deng et al. This is an open access article distributed under the Creative Commons Attribution License, which permits unrestricted use, distribution, and reproduction in any medium, provided the original work is properly cited.

\begin{abstract}
The Stream of Variation (SoV) model and control chart are combined to study the fault diagnosis method of flexible materials R2R manufacturing system. Based on the analysis of the correlation between the fault source and product quality in the manufacturing process and also the statistical distribution rule of the processing quality characteristic vector $L_{i}$ and the fault source $f_{i}$, SoV model under controlled or uncontrolled states and the mathematical model of the probability distribution of the statistic $T_{i, m}^{2}$ of the quality characteristic variable $L_{i}$ are deduced. And the calculation equation of the centerline, the upper limit, and the lower limit of the control chart are deduced. The experimental results show that, under controlled or uncontrolled condition, when the program runs to 500 steps, the Average Run Length (ARL) of the performance parameters tends to be stable; and when program reaches 1000 steps, the actual ARL value is almost the same as the theoretical value. The fault diagnosis experiment shows that, under the condition when the fault source is strongly correlated or the fault source correlation coefficient is the same, using the control chart established in this paper can simply and quickly determine the fault location in the system.
\end{abstract}

\section{Introduction}

$\mathrm{R} 2 \mathrm{R}$ manufacturing system is a typical multistation continuous manufacturing system [1]. Since the factors that affect the quality of $\mathrm{R} 2 \mathrm{R}$ are caused by many related process characteristics such as manufacturing system faults or motion abnormalities, it is difficult for the conventional prediction method to determine the fault source when a manufacturing quality problem occurs. The fault diagnosis method based on the quality control chart classifies the various patterns of control charts from processing quality data, establishes an abnormal pattern set and a fault set, and correlates the abnormal pattern set and the fault set in order to diagnose the fault source of the manufacturing system [2]. The existing quality control chart fault diagnosis methods are univariate control chart [3], multivariate control chart [4], regression adjustment control chart [5], and so on. However, when using these methods to monitor multistation systems, the control charts have a high false alarm rate. In particular, when the process data is autocorrelated, it is impossible to monitor the abnormal faults in the manufacturing process by using conventional control charts under the assumption of independence.

Based on the analysis of the correlation between each station of R2R manufacturing system, combining the physical analysis and data-driven method, this paper establishes the relation equation describing the process deviation of multistation and the final quality of the product, constructs a SoV model under controlled or uncontrolled manufacturing systems, works out corresponding quality control chart for product quality characteristic variables to monitor autocorrelation data, and detects and isolates multiple faults. Hence this paper lays a theoretical foundation for the subsequent intelligent maintenance of R2R manufacturing system. 


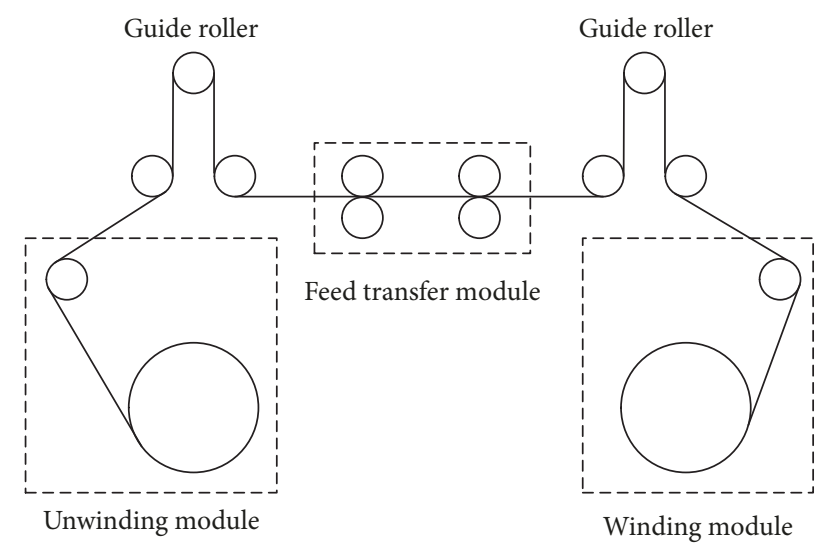

FIGURE 1: A simplified working principle diagram of manufacturing system.

\section{Fault Diagnosis Based on Quality Control Chart}

The flexible material R2R manufacturing system is a continuous multistation manufacturing system as previously described. Figure 1 is a simplified working principle diagram of manufacturing system, the output of the previous station is the input of the next station during manufacturing process, due to incidental factors and system factors, each station output product quality characteristic produces a certain deviation, this deviation then enters the next station, and thus the final quality characteristic deviation of product is the result of gradual accumulation of quality characteristic deviation of all previous stations [6]. Therefore, not only are the system's input and output variables considered, but also the system's real-time statuses are followed in the control of product quality. In order to better analyze the main influencing factors of manufacturing process quality deviation, this paper divides the state of $\mathrm{R} 2 \mathrm{R}$ manufacturing process into controlled state and uncontrolled state. Under the controlled state, it is inevitable that there is a random deviation in manufacturing process, the quality characteristic value shows a certain regularity, and the product quality fluctuation is small. It is difficult to eliminate this deviation, and it is not necessary to eliminate it. Under the uncontrolled state, there are abnormal factors besides the effect of random error in the production process; the values of the quality characteristics fluctuate greatly and have a great impact on the quality. To ensure that the quality maintains the original regularity, the deviation must be eliminated to make production run smoothly [7].

Based on the working principle, as shown in Figure 1, it is possible to judge whether the manufacturing system has abnormal factors by the state of quality control chart of the processing object; Figure 2 shows the basic form of control chart, where the abscissa denotes the number of samples, the ordinate denotes the sample quality characteristic value, UCL denotes upper control limit, CL denotes centerline, LCL denotes lower control limit, quality characteristic values are sampled in chronological order, which are described in the form of a scatter plot in the coordinate system sequentially,

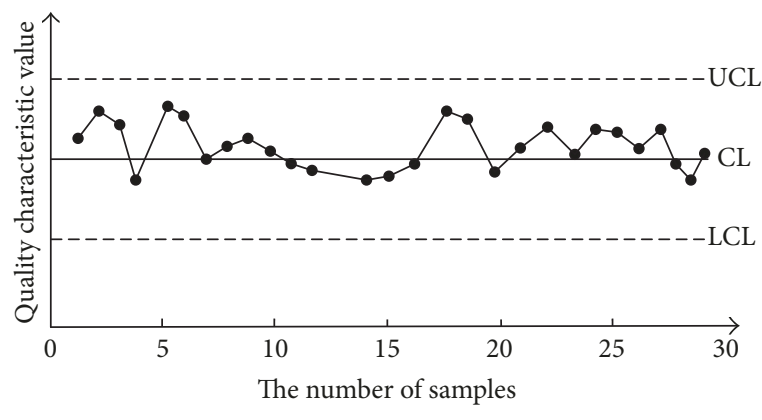

FIgURE 2: The basic form of quality control chart.

and finally the control chart is obtained through the connection to reflect the quality fluctuation in the production process. Under the controlled state, all sample points are between the upper and lower control limits. If the sample points are beyond this limit, then there is an exception or fault during the production process, which means at this moment the system is under an uncontrolled state, and when the data point exceeds the control limit area, an alarm occurs on the control chart.

R2R manufacturing system usually includes hundreds of operations to complete the product processing, many failures of the system may occur at the same time, and the conventional Statistical Process Control (SPC) takes the entire multistation system as a whole; thus it is not able to identify abnormal station. The SoV model based on the physical model of tension transfer can remedy this problem; this method uses the SoV model to obtain the quality data of different workstations to establish the fault diagnosis control chart; after the process change is detected, the fault signal is extracted by means of estimation or pattern matching to determine the root cause so that fault detection and isolation can be achieved.

\section{Construction of the Relationship between Fault Source and Product Quality Model Based on SoV}

If $i$ denotes the $i$ th station, $L_{i}$ is the quality characteristics vector of $N \times 1$-dimensional output product of the $i$ th station (this paper takes the length of the printed image as the quality characteristics), $G$ denotes a constant coefficient matrix determined by a production system, $f_{i}$ is the $N \times 1$ dimensional fault source vector of the $i$ th station, $e_{i}$ is the measurement noise of $N \times 1$ dimension in the $i$ th station and the noise vector which is not included in the model, $\tau$ is constant, and then the SoV basic model that reflects the relationship between fault source and product quality is shown in

$$
L_{i}=\Gamma f_{i}+\varepsilon_{i}
$$

From (1), it is obvious that $L_{i}$, the product quality characteristic, becomes abnormal immediately when the fault source is affected by abnormal factors.

Therefore, the definition in this paper is as follows: if the fault source $f_{i}$ is not affected by abnormal factors, then 
the manufacturing process is under controlled state, and the distribution of the fault source vector obeys $f_{i} \sim N\left(0, \Sigma_{f}\right)\left(\Sigma_{f}\right.$ is the covariance matrix of $f_{i}$ ). The manufacturing process is considered under uncontrolled state if the expectation of fault source $f_{i}$ or the covariance matrix is shifting or if expectation of fault source $f_{i}$ is shifting simultaneously with the covariance matrix. Due to the length limitation of this article, only the anomalies caused by the expected offset of $f_{i}$ are discussed in this paper; at this time, the distribution of fault source vectors obeys $f_{i} \sim N\left(\mu_{f}, \Sigma_{f}\right)(i \geq \tau)$, where $\mu_{f}\left(\mu_{f} \neq 0\right)$ is the mean vector of $f_{i}$ after anomalies occur.

3.1. SoV Model under Controlled State. Because the R2R manufacturing process is affected by the internal system factors, the process output of each station is not independent; that is, the quality data of the previous station affect the quality data of the next station, so that there is autocorrelation among the data.

If $\Phi$ represents the unit diagonal matrix, $v_{i}$ represents a random number matrix that is independent and normal distribution, and the fault source of the current station is linearly related to the fault source of the previous station, then $f_{i}=\Phi f_{i-1}+v_{i}$. Thus assuming $v_{i}$ and $\varepsilon_{i}$ are independent of each other and obey normal distributions $v_{i} \sim N\left(0, \Sigma_{v}\right)$, $\varepsilon_{i} \sim N\left(0, \Sigma_{\varepsilon}\right)$ the distribution of $f_{i}$ obeys $f_{i} \sim N\left(0, \Sigma_{f}\right)$, the distribution of $L_{i}$ obeys $L_{i} \sim N\left(0, \Sigma_{L}\right)$, and the SoV model under the controlled state of R2R manufacturing system is obtained as follows:

$$
\begin{aligned}
L_{i}= & \Gamma f_{i}+\varepsilon_{i} \\
f_{i}= & \Phi f_{i-1}+v_{i} \\
\Sigma_{L}= & \Gamma \Phi^{i} \Sigma_{f}\left(\Gamma \Phi^{i}\right)^{\prime}+\Gamma \Phi^{i-1} \Sigma_{v}\left(\Gamma \Phi^{i-1}\right)^{\prime}+\cdots \\
& +\Gamma \Phi \Sigma_{v}(\Gamma \Phi)^{\prime}+\Gamma \Sigma_{v}(\Gamma)^{\prime}+\Sigma_{\varepsilon} .
\end{aligned}
$$

Further derivation is as follows:

$$
\begin{aligned}
& L_{i}=\Gamma f_{i}+\varepsilon_{i} \\
&=\Gamma\left(\Phi f_{i-1}+v_{i}\right)+\varepsilon_{i} \\
&=\Gamma \Phi f_{i-1}+\Gamma v_{i}+\varepsilon_{i} \\
&=\Gamma \Phi\left(\Phi f_{i-2}+v_{i-1}\right)+\Gamma v_{i}+\varepsilon_{i} \\
&=\Gamma \Phi^{2} f_{i-2}+\Gamma \Phi v_{i-1}+\Gamma v_{i}+\varepsilon_{i} \\
& \quad \vdots \\
&=\Gamma \Phi^{i} f_{0}+\Gamma \Phi^{i-1} f_{1}+\Gamma \Phi^{i-2} f_{2}+\cdots+\Gamma \Phi v_{i-1}+\Gamma v_{i} \\
&+\varepsilon_{i} .
\end{aligned}
$$

In the equation above, the diagonal matrix $\Phi$ indicates the relevant level of the fault source. In general, $\Phi=\phi I(-1<$ $\phi<1)$ and $I$ is the unit matrix.
3.2. SoV Model under Uncontrolled State. When one station of R2R manufacturing system malfunctions, the expected value of $f_{i}$ shifts. If $\mu_{f}$ is the mean vector after the change of fault source, $\Sigma_{f}$ is covariance matrix of fault source, offset direction of $f_{i}$ is $d_{f}$, and offset is $\delta$, then the offset of $f_{i}$ is $\mu_{f}=\delta d_{f}$, and the distribution of $f_{i}$ changes from $f_{i} \sim$ $N\left(0, \Sigma_{f}\right)$ to $f_{i} \sim N\left(\mu_{f}, \Sigma_{f}\right)$. Thus $\mu_{L}$ represents the mean of $L_{i}, \Sigma_{L}$ represents the covariance of $L_{i}$, and $L_{i}$ obeys the distribution $L_{i} \sim N\left(\mu_{L}, \Sigma_{L}\right)$; then the SoV model under the uncontrolled state of the $\mathrm{R} 2 \mathrm{R}$ manufacturing process is as follows:

$$
\begin{aligned}
L_{i}= & \Gamma\left(f_{i}+\mu_{f}\right)+\varepsilon_{i} \\
\mu_{L}= & +\Gamma \Phi^{i-\tau} \mu+\Gamma \Phi^{i-(\tau+1)} \mu+\cdots+\Gamma \Phi^{2} \mu+\Gamma \Phi \mu+\Gamma \mu \\
\Sigma_{L}= & \Gamma \Gamma \Phi^{i-\tau} \Sigma_{f}\left(\Gamma \Phi^{i-\tau}\right)^{\prime}+\Gamma \Phi^{i-(\tau+1)} \Sigma_{v}\left(\Gamma \Phi^{i-(\tau+1)}\right)^{\prime} \\
& +\cdots+\Gamma \Phi \Sigma_{v}(\Gamma \Phi)^{\prime}+\Gamma \Sigma_{v}(\Gamma)^{\prime}+\Sigma_{\varepsilon} \\
= & {\left[\phi^{2}\right]^{i-\tau} \Gamma \Gamma^{\prime}+\left[\phi^{2}\right]^{i-(\tau+1)} \Gamma \Gamma^{\prime}+\cdots+\phi^{2} \Gamma \Gamma^{\prime}+\Gamma \Gamma^{\prime} } \\
& +I=\frac{1-\phi^{2}}{1-\phi^{2(i-\tau+1)}} \Gamma \Gamma^{\prime}+I .
\end{aligned}
$$

Further derivation is as follows:

$$
\begin{aligned}
& L_{i}=\Gamma\left(f_{i}+\mu\right)+\varepsilon_{i} \\
&=\Gamma\left(\Phi\left(f_{i-1}+\mu\right)+v_{i}\right)+\Gamma \mu+\varepsilon_{i} \\
&=\Gamma \Phi f_{i-1}+\Gamma v_{i}+\Gamma \Phi \mu+\Gamma \mu+\varepsilon_{i} \\
&=\Gamma \Phi\left(\Phi\left(f_{i-2}+\mu\right)+v_{i-1}\right)+\Gamma v_{i}+\Gamma \Phi \mu+\Gamma \mu+\varepsilon_{i} \\
&=\Gamma \Phi^{2} f_{i-2}+\Gamma \Phi v_{i-1}+\Gamma v_{i}+\Gamma \Phi \mu+\Gamma \Phi^{2} \mu+\Gamma \mu+\varepsilon_{i} \\
& \quad \vdots \\
&=\Gamma \Phi^{i-\tau} f_{\tau}+\Gamma \Phi^{i-(\tau+1)} v_{\tau+1}+\Gamma \Phi^{i-(\tau+2)} v_{\tau+2}+\cdots \\
&+\Gamma \Phi v_{i-1}+\Gamma v_{i}+\varepsilon_{i}+\Gamma \Phi^{i-\tau} \mu+\Gamma \Phi^{i-(\tau+1)} \mu+\cdots \\
&+\Gamma \Phi^{2} \mu+\Gamma \Phi \mu+\Gamma \mu .
\end{aligned}
$$

So far, the SoV model that expresses the relationship between the fault source in manufacturing process and the final quality of the product under both the controlled and the uncontrolled states of the R2R manufacturing system has been derived. The next section will establish a control chart for monitoring the autocorrelation data during the R2R manufacturing process, according to the distribution of the statistic $T_{i, m}^{2}$ of the quality characteristic variable $L_{i}$.

\section{Based on the Distribution of Statistic $T_{i, m}^{2}$ to Establish Fault Source Control Chart}

In previous session, SoV model of R2R manufacturing system under controlled state and uncontrolled state is discussed; this session sets up the control chart of the corresponding fault source for the statistical distribution of $L_{i}$. 
4.1. The Distribution Mathematical Expression Derivation of Statistic $T_{i, m}^{2}$. When the mean value of the fault source $f_{i}$ shifts, the mean value of the corresponding $L_{i}$ shifts, and each offset direction of $f_{i}$ corresponds to an offset direction of $L_{i}$. It is assumed that a total of $t$ stations have failure, each station corresponds to an offset direction $\delta$, the system has a total of $t$ offset directions $\rho_{m}\left(m=1,2, \ldots, t,\left|\rho_{m}\right|=1\right)$, and then average offset of $f_{i}$ is $\mu_{f, m}=\delta \rho_{m}$.

When $i=\tau$, a station failure of R2R manufacturing system occurs, the mean of $f_{i}$ shifts, and the change direction of $L_{i}$ can be calculated by the mean change direction of $f_{i}$ with reference to (4); that is, the updated quality characteristic vector $L_{i, m}$ is obtained:

$$
\begin{aligned}
L_{i, m}= & \Gamma\left(f_{i}+\mu_{f, m}\right)+\varepsilon_{i} \\
= & \Gamma \Phi^{i-\tau} f_{\tau}+\Gamma \Phi^{i-(\tau+1)} v_{\tau+1}+\Gamma \Phi^{i-(\tau+2)} v_{\tau+2}+\cdots \\
& +\Gamma \Phi v_{i-1}+\Gamma v_{i}+\varepsilon_{i}+\Gamma \Phi^{i-\tau} \mu_{f, m} \\
& +\Gamma \Phi^{i-(\tau+1)} \mu_{f, m}+\cdots+\Gamma \Phi^{2} \mu_{f, m}+\Gamma \Phi \mu_{f, m} \\
& +\Gamma \mu_{f, m} .
\end{aligned}
$$

Obviously, the mean change of $L_{i, m}$ is as follows:

$$
\begin{aligned}
h_{L, m}= & \Gamma \Phi^{i-\tau} \mu_{f, m}+\Gamma \Phi^{i-(\tau+1)} \mu_{f, m}+\cdots+\Gamma \Phi^{2} \mu_{f, m} \\
& +\Gamma \Phi \mu_{f, m}+\Gamma \mu_{f, m} .
\end{aligned}
$$

Further, the resulting direction vector $d_{L, m}$ of $h_{L, m}$ is

$$
\begin{aligned}
& d_{L, m}=\frac{h_{L, m}}{\left|h_{L, m}\right|} \\
& =\frac{\Gamma \Phi^{i-\tau} \mu_{f, m}+\Gamma \Phi^{i-(\tau+1)} \mu_{f, m}+\cdots+\Gamma \Phi^{2} \mu_{f, m}+\Gamma \Phi \mu_{f, m}+\Gamma \mu_{f, m}}{\left|\Gamma \Phi^{i-\tau} \mu_{f, m}+\Gamma \Phi^{i-(\tau+1)} \mu_{f, m}+\cdots+\Gamma \Phi^{2} \mu_{f, m}+\Gamma \Phi \mu_{f, m}+\Gamma \mu_{f, m}\right|} \\
& =\frac{\Gamma\left(\phi^{i-\tau}+\phi^{i-(\tau+1)}+\cdots+\phi^{2}+\phi+1\right) I \mu_{f, m}}{\left|\Gamma\left(\phi^{i-\tau}+\phi^{i-(\tau+1)}+\cdots+\phi^{2}+\phi+1\right) I \mu_{f, m}\right|}=\frac{\Gamma \rho_{m}}{\left|\Gamma \rho_{m}\right|} .
\end{aligned}
$$

From (8), $d_{L, m}$ does not relate to $i$ nor $\tau$; it relates to $\Gamma$ and $\mu_{f, m}$; when designing control charts with statistic, according to Hawkins's proof [8], the statistic shown in (9) is the most effective:

$$
T_{i, m}^{2}=d_{L, m}^{\prime} \sum_{L}^{-1} L_{i}
$$

Because $L_{i}$ obeys distribution $L_{i} \sim N\left(\mu_{L}, \Sigma_{L}\right)$, the mean vector and the covariance matrix are calculated separately, and the distribution of the statistic $T_{i, m}^{2}$ is obtained as follows:

$$
T_{i, m}^{2} \sim N\left(d_{L, m}^{\prime} \sum_{L}^{-1} \mu_{L}, d_{L, m}^{\prime} \sum_{L}^{-1} d_{L, m}\right), \quad m=1, \ldots, t .
$$

Based on the distribution mathematical expression of statistic $T_{i, m}^{2}$, the equation of control centerline, upper limit, and lower limit under controlled or uncontrolled state in control chart is going to be derived.
4.2. The Equation Derivation of Centerline, the Upper Control Limit, and the Lower Control Limit in Control Chart. (1) When $i<\tau$ system is under controlled state, $f_{i}$ and $L_{i}$ obey the distributions $f_{i} \sim N\left(0, \Sigma_{f}\right), L_{i} \sim N\left(0, \Sigma_{L}\right)$, and then the distribution of statistic $T_{i, m}^{2}$ is

$$
T_{i, m}^{2} \sim N\left(0, d_{L, m}^{\prime} \sum_{L}^{-1} d_{L, m}\right)
$$

Since $f_{i}$ has $t$ possible directions of change, so $t$ pieces of control chart are needed to monitor the changes of $f_{i}$; if there is an alarm in a control chart, it indicates that the manufacturing process is under uncontrolled state. In order to establish Shewhart control charts $[9,10]$ for $T_{i, m}^{2}$, the centerline, upper control limit, and lower control limit of each control chart are shown in

$$
\begin{gathered}
\mathrm{CL}_{m}=d_{L, m}^{\prime} \sum_{L}^{-1} \mu_{L}=0 \\
\mathrm{UCL}_{m}=d_{L, m}^{\prime} \sum_{L}^{-1} \mu_{L}+A \sigma_{T_{i, m}^{2}}=A \sigma_{T_{i, m}^{2}} \\
\mathrm{LCL}_{m}=d_{L, m}^{\prime} \sum_{L}^{-1} \mu_{L}-A \sigma_{T_{i, m}^{2}}=-A \sigma_{T_{i, m}^{2}},
\end{gathered}
$$

where $m=1,2, \ldots, t, A$ is control limit factor, and $\sigma_{T_{i, m}^{2}}$ is the standard deviation of statistic $T_{i, m}^{2}$.

(2) When $i=\tau$ the fault has just started and $f_{i}$ obeys the distribution $f_{i} \sim N\left(\mu_{f}, \Sigma_{f}\right)$, then the distribution of statistic $T_{i, m}^{2}$ is shown in

$$
T_{i, m}^{2} \sim N\left(d_{L, m}^{\prime} \sum_{L}^{-1}\left(\Gamma \mu_{f}\right), d_{L, m}^{\prime} \sum_{L}^{-1} d_{L, m}\right) .
$$

The centerline, the upper control limit, and the lower control limit of each control chart are shown in

$$
\begin{aligned}
\mathrm{CL}_{m} & =d_{L, M}^{\prime} \sum_{L}^{-1} \mu_{L}=d_{L, M}^{\prime} \sum_{L}^{-1}\left(\Gamma \mu_{f}\right) \\
\mathrm{UCL}_{m} & =d_{L, M}^{\prime} \sum_{L}^{-1} \mu_{L}+A \sigma_{T_{i, m}^{2}}=d_{L, M}^{\prime} \sum_{L}^{-1}\left(\Gamma \mu_{f}\right)+A \sigma_{T_{i, m}^{2}} \\
\mathrm{LCL}_{m} & =d_{L, M}^{\prime} \sum_{L}^{-1} \mu_{L}-A \sigma_{T_{i, m}^{2}} \\
& =d_{L, M}^{\prime} \sum_{L}^{-1}\left(\Gamma \mu_{f}\right)-A \sigma_{T_{i, m}^{2}} .
\end{aligned}
$$


(3) When $i>\tau$ the fault continues to occur and $f_{i}$ obeys the distribution $f_{i} \sim N\left(\mu_{f}, \Sigma_{f}\right)$, then the distribution of statistic $T_{i, m}^{2}$ is shown in

$$
\begin{aligned}
T_{i, m}^{2} & \sim N\left(d _ { L , m } ^ { \prime } \sum _ { L } ^ { - 1 } \left(\Gamma \Phi^{i-\tau} \mu_{f, m}+\Gamma \Phi^{i-(\tau+1)} \mu_{f, m}+\cdots\right.\right. \\
& \left.\left.+\Gamma \Phi \mu_{f, m}+\Gamma \mu_{f, m}\right), d_{L, m}^{\prime} \sum_{L}^{-1} d_{L, m}\right) .
\end{aligned}
$$

Hence the centerline, the upper control limit, and the lower control limit of each control chart are shown in

$$
\begin{aligned}
& \mathrm{CL}_{m}=d_{L, M}^{\prime} \sum_{L}^{-1}\left(\Gamma \Phi^{i-\tau} \mu_{f, m}+\Gamma \Phi^{i-(\tau+1)} \mu_{f, m}+\cdots\right. \\
& \left.+\Gamma \Phi \mu_{f, m}+\Gamma \mu_{f, m}\right) \\
& \mathrm{UCL}_{m}=d_{L, M}^{\prime} \sum_{L}^{-1}\left(\Gamma \Phi^{i-\tau} \mu_{f, m}+\Gamma \Phi^{i-(\tau+1)} \mu_{f, m}+\cdots\right. \\
& \left.+\Gamma \Phi \mu_{f, m}+\Gamma \mu_{f, m}\right)+A \sigma \\
& \mathrm{LCL}_{m}=d_{L, M}^{\prime} \sum_{L}^{-1}\left(\Gamma \Phi^{i-\tau} \mu_{f, m}+\Gamma \Phi^{i-(\tau+1)} \mu_{f, m}+\cdots\right. \\
& \left.+\Gamma \Phi \mu_{f, m}+\Gamma \mu_{f, m}\right)-A \sigma .
\end{aligned}
$$

The coordinate system is established on the centerline, the upper control limit, and the lower control limit calculated according to (12), (14), and (16), respectively, and the manufacturing process data are collected and traced in the coordinate system; then the control chart design is completed.

\section{Verification Experiment}

5.1. Control Chart Performance Verification Experiment. ARL is used as a measure of control chart performance [11, 12]. ARL of control chart under controlled state $\mathrm{ARL}_{\text {in }}=$ $\sum_{i=1}^{\infty} i\left(1-p_{1}\right)^{i-1} p_{1}=1 / p_{1}$, where $p_{1}$ is alarm probability of the $t$ th control chart; ARL of control chart under uncontrolled state $\mathrm{ARL}_{\text {out }}=1 \times p_{2}+\left(1-p_{2}\right) \sum_{i=2}^{\infty} i\left(1-p_{3}\right)^{i-2} p_{3}=$ $\left(1-p_{2}+p_{3}\right) / p_{3}$, where $p_{2}$ indicates the alarm probability of each control chart at the beginning of the fault when $i=\tau$, $p_{3}$ indicates probability of alarm of each control chart when $i>\tau$, the fault continues to occur, and the mean value of the fault source $f$ changes while the covariance matrix does not change.

There are 4 directions of fault source $(T=4)$; using the Mento Carlo method to simulate the autocorrelation data of multistation manufacturing process [13], the validity of the proposed control chart is verified.

(1) Taking control coefficient $A=3$ and according to $3 \sigma$ principle, the average length of the operation under controlled state is calculated as $\mathrm{ARL}_{\text {in }}=1 / p_{1}=370.40, \rho, \Gamma$, $\phi$ are randomly generated by computer numerical simulation

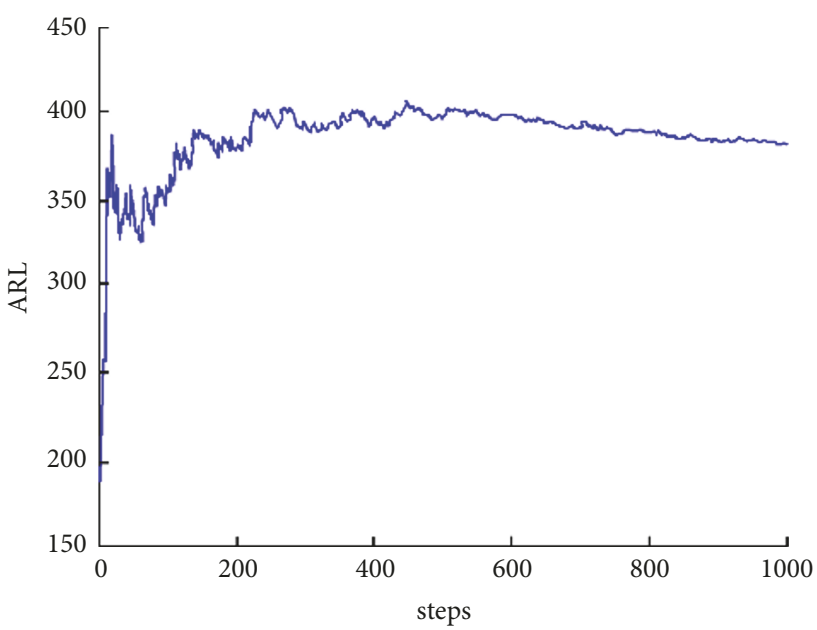

FIGURE 3: Distribution of $\mathrm{ARL}_{\text {in }}$ under controlled state.

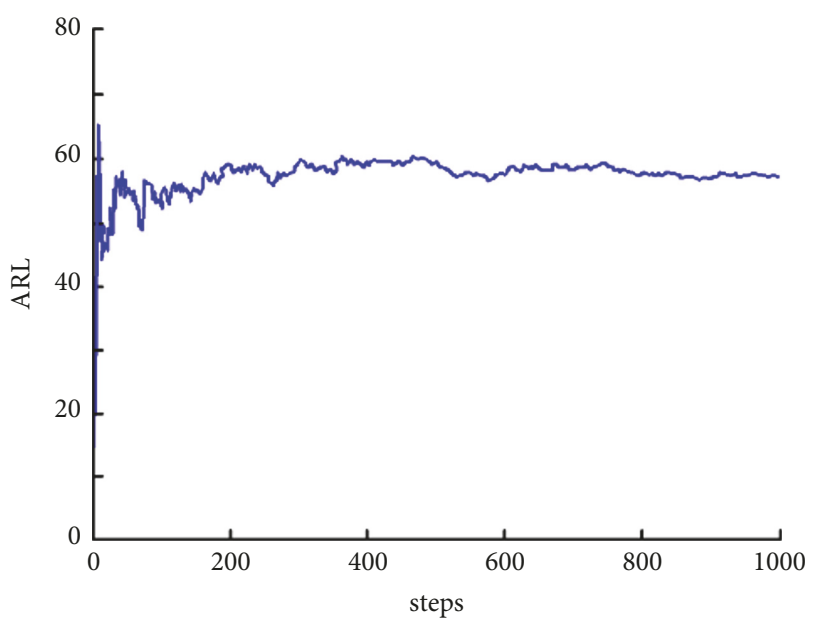

FiguRE 4: Distribution of $\mathrm{ARL}_{\text {in }}$ under uncontrolled state.

software, wherein $\phi \in(0,1), \Gamma$ is $t \times t$ dimension, $\rho$ is $t \times 1$ dimension, and the result data are as follows: $\phi=0.4, \rho=$ $\left(\begin{array}{llll}0.0856 & -0.2793 & 0.8457 & 0.3587\end{array}\right)^{T}$ :

$$
\Gamma=\left(\begin{array}{cccc}
0.7256 & -1.5284 & 1.2148 & -0.8369 \\
1.5243 & 1.3692 & -0.7648 & 0.8052 \\
0.2968 & 1.2658 & -0.6839 & 1.1583 \\
-0.9254 & -0.4835 & 1.0258 & 0.4236
\end{array}\right) \text {. }
$$

The distribution of $A R L_{\text {in }}$ in controlled state shown in Figure 3 is further obtained.

As analysis from Figure 3, when the program runs to 1000 steps, $\mathrm{ARL}_{\text {in }}=369.254$, which is basically the same as its theoretical value. Although the previous period fluctuates greatly, the program becomes stable after 500 steps.

(2) Using the above randomly generated $\rho, \Gamma, \phi$, if the expected length of change in size $\delta=2$, then $\mu_{f}=$ $2 \rho$. At this moment, multistation manufacturing process is under uncontrolled state; that is, a fault has occurred; after calculating $\mathrm{ARL}_{\text {out }}=\left(1-p_{2}+p_{3}\right) / p_{3}=56.87$, the $\mathrm{ARL}_{\text {out }}$ distribution chart under uncontrolled state shown in Figure 4 is obtained. 


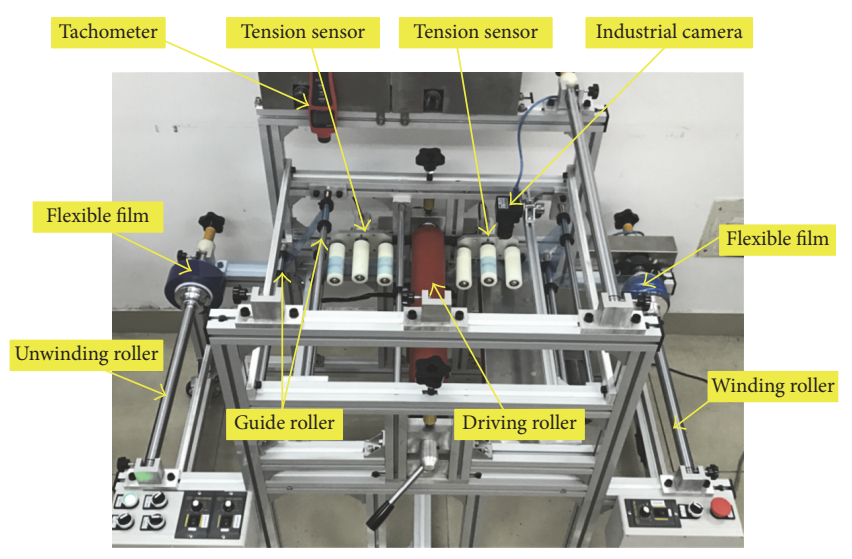

FIGURE 5: R2R manufacturing unwinding-conveyor-winding experimental device.

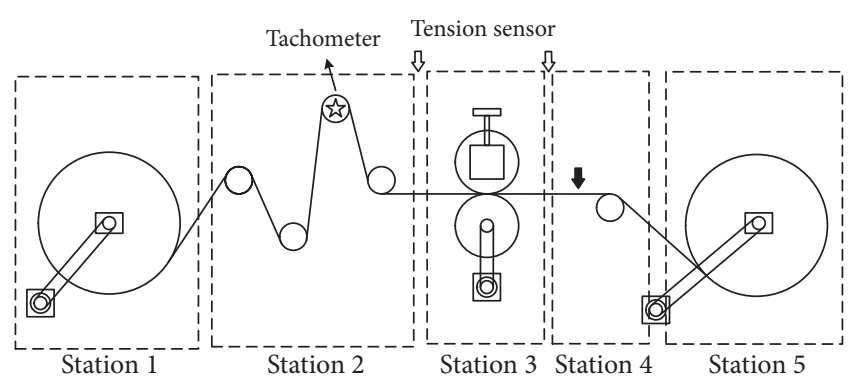

FIGURE 6: Experimental platform working principle diagram.

As Figure 4, when the program runs to step 1000, $\mathrm{ARL}_{\text {out }}=57.48$ which is relatively close to theoretical value. Similar to the above, the previous period fluctuates greatly, and the program becomes stable after 500 steps.

\subsection{R2R Manufacturing System Fault Diagnosis Experi-} ment. The flexible material R2R manufacturing experimental equipment shown in Figure 5 is built for fault prediction test; the working principle of the experimental equipment is shown in Figure 6. This equipment contains unwinding roller module, winding roller module, guide roller module, and driving roller module and each module adopts $120 \mathrm{~W}$ speed motor. The driving module adopts the counter-roller mode, the rubber roller is driven by the motor to rotate, the rubber roller is controlled by a hand-held lifting handle, and the upper and lower rollers rotate at the same time to realize material transmission. The drive module adopts the symmetrical roller mode: motor drives lower rubber roller to rotate and upper rubber roller is controlled by a hand-held lifting handle. First, lifting the upper rubber roller, the material is flattened after being placed around the roller, and then after accurate alignment the rubber roller is put down. Finally, the upper and lower rollers start to rotate at the same time to realize material transmission. The maximum adaptation width of the experimental platform is $450 \mathrm{~mm}$, which can be used to transfer material thickness of $0.1 \mathrm{~mm}-5 \mathrm{~mm}$. Blue PET polyester film is selected in this experiment; the details of this material are as follows: width is
$50 \mathrm{~mm}$, thickness is $0.05 \mathrm{~mm}$, density is $1450 \mathrm{~kg} / \mathrm{m}^{3}$, modulus of elasticity is $3495 \mathrm{MPa}$, and Poisson's ratio is 0.3 .

(1) Unwinding process station number $N=4$, the unwinding process affected by faults in four directions is defined, and a control chart of the autocorrelation data monitoring during the unwinding process is established.

(1) Combining the SoV model of R2R unwinding process for flexible material given in reference [14] and (2), the coefficient matrix of unwinding process $\Gamma$ is calculated:

$$
\Gamma=\left(\begin{array}{cccc}
1.0000 & 0 & 0 & 0 \\
1.0258 & 1.0000 & 0 & 0 \\
1.1056 & 0.9583 & 1.0000 & 0 \\
1.1947 & 1.1056 & 1.0258 & 1.0000
\end{array}\right)
$$

(2) Considering the four-station situation, the direction of the fault and offset direction of $L_{i}$ are determined: $\rho_{1}=$ $(1,0,0,0)^{T}, \rho_{2}=(0,1,0,0)^{T}, \rho_{3}=(0,0,1,0)^{T}$, and $\rho_{4}=$ $(0,0,0,1)^{T}$.

(3) From (8), $d_{L, m}=\Gamma \rho_{m} /\left|\Gamma \rho_{m}\right|, d_{L, m}$ is calculated, according to the statistic mentioned in (9), $\mathrm{CL}_{m}, \mathrm{UCL}_{m}$, and $\mathrm{LCL}_{m}$ of the control chart are determined, and the data are obtained in Table 1.

(4) According to $\mathrm{CL}_{m}, \mathrm{UCL}_{m}$, and $\mathrm{LCL}_{m}$ of the control chart, the output $L_{i}$ of each station in the unwinding process is monitored. When the data point exceeds the control limit area, the control chart will create alarm.

(5) The theoretical and measured values of $\mathrm{ARL}_{\text {in }}$ and $\mathrm{ARL}_{\text {out }}$ are calculated by the computer numerical simulation software when calculating different values of $\phi$ and $\rho_{m}$. From the previous section, it is known that $\mathrm{ARL}_{\text {in }}=370.40$, and its actual values are shown in Table 2. It is clear that the characteristics of the control chart at this moment are basically consistent with the conclusions obtained in the previous simulation analysis, thereby verifying the effectiveness of the control chart.

(6) For the unwinding process under uncontrolled state, the unwinding process is supposed to be out of control at the beginning. The ARL of the control chart is shown in Figure 6 under different correlation coefficient $\phi$ and fault source offset $\delta$.

From Figure 7, it is obvious that when the fault source offset is the same, the larger the fault source correlation coefficient is, the larger the ARL of the control graph will be. When the fault source has weak correlation, the performance of control chart is very good; however, when the fault source has a strong correlation, although the performance of the control chart decreases slightly, an alarm will occur quickly. With the same correlation coefficient, the larger the fault offset is, the faster the control chart will create alarm. As a result, which station has failed can be determined and staff members can obtain reference information without checking on the stations one by one.

\section{Conclusion}

The product quality of the R2R manufacturing process not only relates to the input and output variables of the system, 
TABLE 1: The calculated values of control limits for each fault direction.

\begin{tabular}{|c|c|c|c|c|c|}
\hline Station number & $\rho_{m}$ & $d_{L, m}$ & $\mathrm{CL}_{m}$ & $\mathrm{UCL}_{m}$ & $\mathrm{LCL}_{m}$ \\
\hline 1 & $(1,0,0,0)^{T}$ & $(0.4612,0.4731,0.5099,0.5510)^{T}$ & 0 & 1.059 & -1.059 \\
\hline 2 & $(0,1,0,0)^{T}$ & $(0,0.5643,0.5407,0.6239)^{T}$ & 0 & 1.153 & -1.153 \\
\hline 3 & $(0,0,1,0)^{T}$ & $(0,0,0.6980,0.7161)^{T}$ & 0 & 1.428 & -1.428 \\
\hline 4 & $(0,0,0,1)^{T}$ & $(0,0,0,1)^{T}$ & 0 & 1.736 & -1.736 \\
\hline
\end{tabular}

TABLE 2: The actual value of $\mathrm{ARL}_{\text {in }}$ when the program runs to 1000 steps under controlled state and different values of $\phi$ and $\rho_{m}$.

\begin{tabular}{ccccccccc}
\hline$\rho_{m}$ & & & & \multicolumn{3}{c}{$\phi$} & & \\
& -0.7 & -0.5 & -0.3 & -0.1 & 0.1 & 0.3 & 0.5 \\
\hline$\rho_{1}$ & 365.84 & 368.84 & 376.02 & 371.35 & 369.07 & 373.56 & 365.93 & 369.24 \\
$\rho_{2}$ & 376.92 & 368.24 & 369.02 & 367.45 & 368.85 & 371.75 & 370.86 & 374.24 \\
$\rho_{3}$ & 369.57 & 368.56 & 368.85 & 365.47 & 373.46 & 370.75 & 369.34 & 371.43 \\
$\rho_{4}$ & 368.30 & 369.43 & 372.46 & 371.53 & 370.29 & 369.24 & 369.74 \\
\hline
\end{tabular}

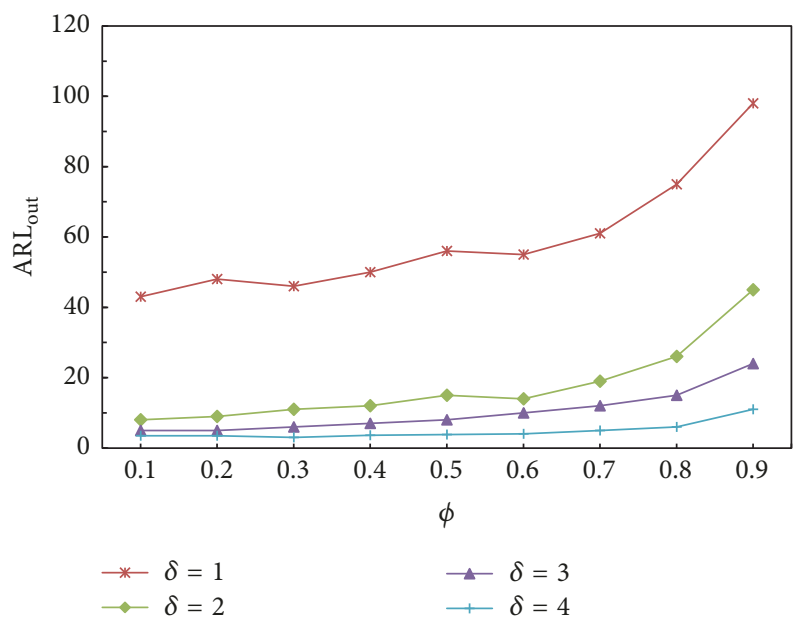

FIGURE 7: $\mathrm{ARL}_{\text {out }}$ under different values of $\phi$ and $\delta$.

but also relates to the real-time status of the system. This paper combines the theoretical method of SoV and control chart and obtains quality data from different stations based on SoV model to establish the control chart of system fault diagnosis.

The length $L_{i}$ of printed image is used as a quality characteristic. $f_{i}$ denotes the fault source vector of a station, $i$ denotes the measurement noise and the noise vector which is not included in the model, and the SoV basic model reflecting the relationship between the fault source and the product quality of the manufacturing process is established as $L_{i}=$ $\Gamma f_{i}+\varepsilon_{i}$. Combining the statistical distribution rules of $L_{i}$ and $f_{i}$ under controlled state and uncontrolled state, the basic model is expanded, respectively; finally the probability distribution $T_{i, m}^{2}$ of $L_{i}$ 's statistic is obtained. With reference to this, the calculation equation of the centerline, upper limit, and lower limit of the control chart are deduced.

The ARL is used as a measure of the control chart performance to conduct the verification experiment. The results show that the actual value of the ARL is basically the same as the theoretical value when the program runs to 1000 steps under controlled or uncontrolled state. Although the fluctuations in the previous period are large, they become stable after 500 steps. The fault diagnosis experiment of R2R manufacturing system shows that when the fault source has a weak correlation, the control chart performance is very good; when the fault source has a strong correlation, although the control chart performance decreases slightly, the alarm occurs quickly; when the correlation coefficient of the fault source is the same, the greater the offset of the fault source is, the faster the control chart creates alarm. As a result, it is simple and fast to locate the system station which has a failure.

\section{Data Availability}

The data used to support the findings of this study are available from the corresponding author upon request.

\section{Conflicts of Interest}

The authors declared no potential conflicts of interest with respect to the research, authorship, and/or publication of this article.

\section{Acknowledgments}

The authors disclosed receipt of the following financial support for the research, authorship, and/or publication of this article. This work was supported in part by the National Natural Science Foundation of China under Grant no. 51675109, Natural Science Foundation of Guangdong Province, China, under Grant no. 2017A030313308, and Provincial Science and Technology Plan Project of Guangdong Province, China, under Grant no. 2016B010124002 and in part by the Provincial Science and Technology Plan of Guangdong Province, China, under Grant no. 2017B010117011 and Grant no. 2016B010112003.

\section{References}

[1] Y. Deng, X. Liu, Z. Zheng, Q. Zhang, and L. Wu, "A new active contour modeling method for processing-path extraction of 
flexible material," Optik - International Journal for Light and Electron Optics, vol. 127, no. 13, pp. 5422-5429, 2016.

[2] L. Rajaoarisoa and M. Sayed-Mouchaweh, "Adaptive online fault diagnosis of manufacturing systems based on DEVS formalism," IFAC-PapersOnLine, vol. 50, no. 1, pp. 6825-6830, 2017.

[3] C. Yiakopoulos, M. Koutsoudaki, K. Gryllias, and I. Antoniadis, "Improving the performance of univariate control charts for abnormal detection and classification," Mechanical Systems and Signal Processing, vol. 86, pp. 122-150, 2017.

[4] S. Ahmad, S. A. Abbasi, M. Riaz, and N. Abbas, "On efficient use of auxiliary information for control charting in SPC," Computers \& Industrial Engineering, vol. 67, no. 1, pp. 173-184, 2014.

[5] X. Dang, L. Yan, H. Jiang, X. Wu, and H. Sun, "Open-circuit voltage-based state of charge estimation of lithium-ion power battery by combining controlled auto-regressive and moving average modeling with feedforward-feedback compensation method," International Journal of Electrical Power \& Energy Systems, vol. 90, pp. 27-36, 2017.

[6] Y. Jiao and D. Djurdjanovic, "Compensability of errors in product quality in multistage manufacturing processes," Journal of Manufacturing Systems, vol. 30, no. 4, pp. 204-213, 2011.

[7] P. R. Raul and P. R. Pagilla, "Design and implementation of adaptive PI control schemes for web tension control in roll-toroll (R2R) manufacturing," ISA Transactions ${ }^{\circledR}$, vol. 56, pp. 276287, 2015.

[8] Z. T. Kosztyán and A. I. Katona, "Risk-based multivariate control chart," Expert Systems with Applications, vol. 62, pp. 250-262, 2016.

[9] G. Verdier, "Application of copulas to multivariate control charts," Journal of Statistical Planning and Inference, vol. 143, no. 12, pp. 2151-2159, 2013.

[10] A. Khormali and J. Addeh, "A novel approach for recognition of control chart patterns: Type-2 fuzzy clustering optimized support vector machine," ISA Transactions ${ }^{\circledR}$, vol. 63, pp. 256264, 2016.

[11] J. Yue and L. Liu, "Multivariate nonparametric control chart with variable sampling interval," Applied Mathematical Modelling: Simulation and Computation for Engineering and Environmental Systems, vol. 52, pp. 603-612, 2017.

[12] M. A. Graham, A. Mukherjee, and S. Chakraborti, "Distribution-free exponentially weighted moving average control charts for monitoring unknown location," Computational Statistics \& Data Analysis, vol. 56, no. 8, pp. 2539-2561, 2012.

[13] Y. Liu, M. Yuan, J. Cao, J. Cui, and J. Tan, "Evaluation of measurement uncertainty in $\mathrm{H}$-drive stage during high acceleration based on Monte Carlo method," The International Journal of Machine Tools and Manufacture, vol. 93, article no. 3040, pp. 1-9, 2015.

[14] H. Tang, J.-A. Duan, S. Lan, and H. Shui, "A new geometric error modeling approach for multi-axis system based on stream of variation theory," The International Journal of Machine Tools and Manufacture, vol. 92, pp. 41-51, 2015. 


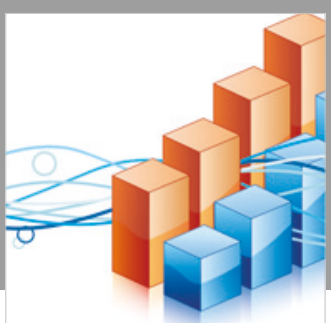

Advances in

Operations Research

\section{-n-m}
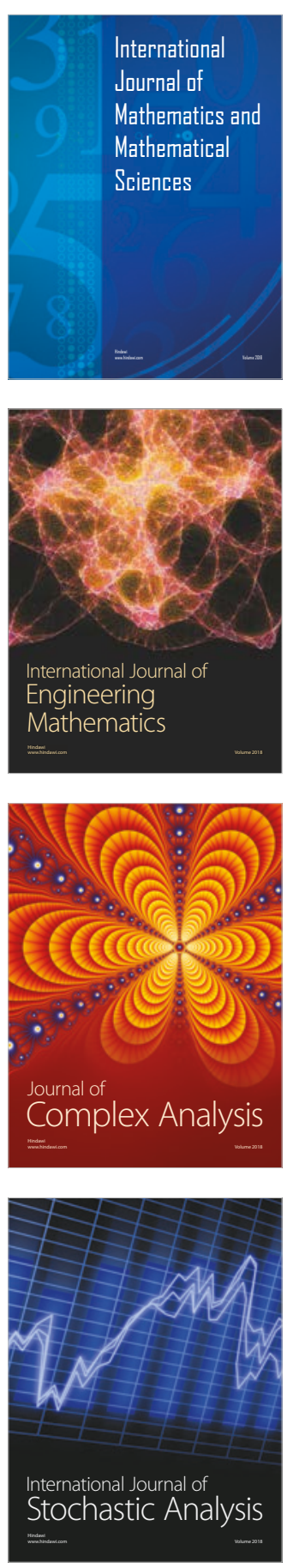
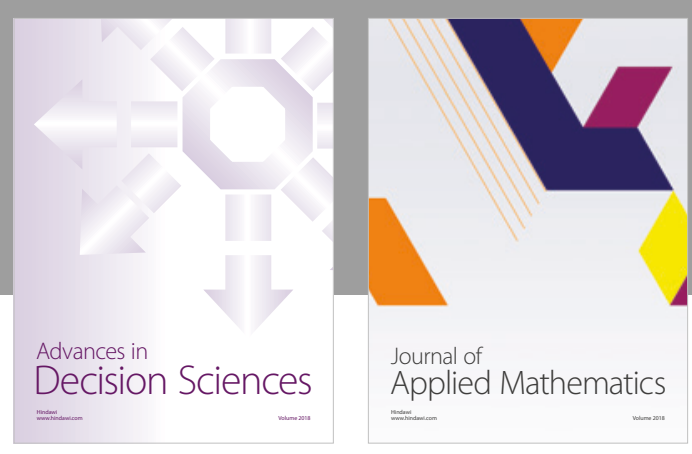

Journal of

Applied Mathematics
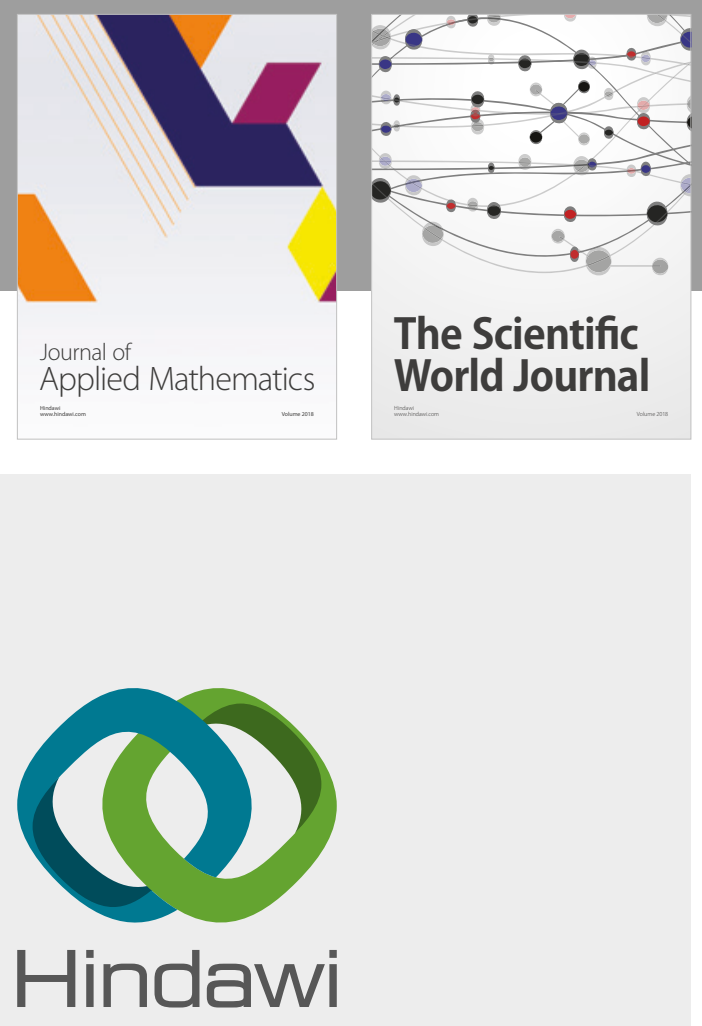

Submit your manuscripts at

www.hindawi.com

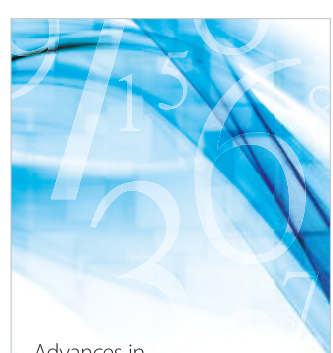

Advances in
Numerical Analysis
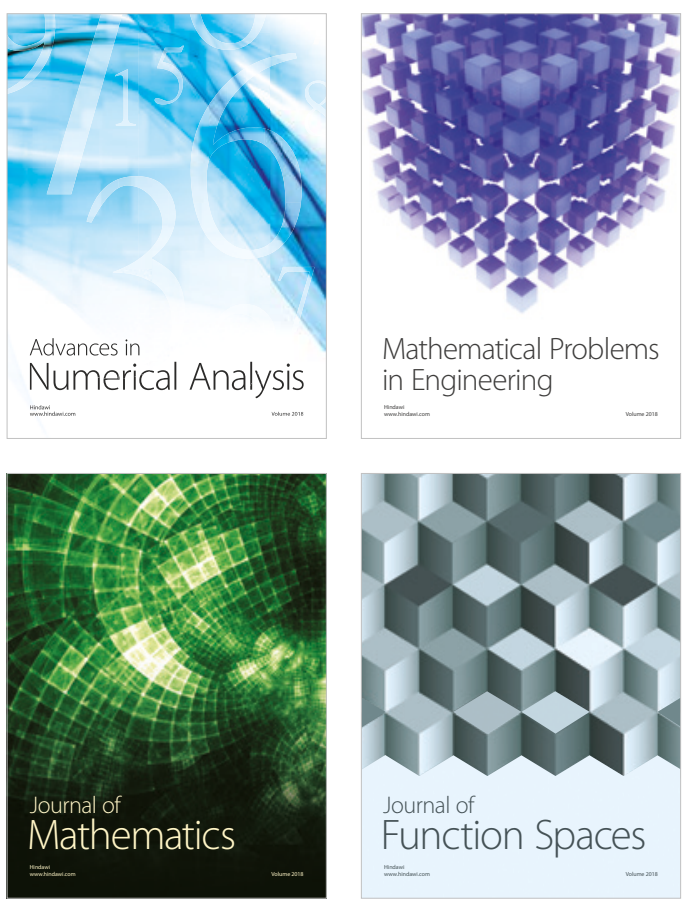

Mathematical Problems in Engineering

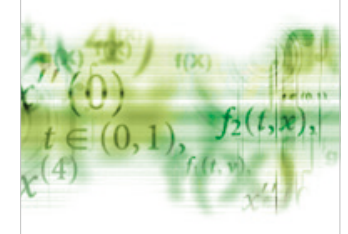

International Journal of

Differential Equations

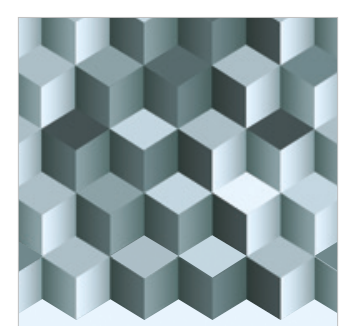

Journal of

Function Spaces

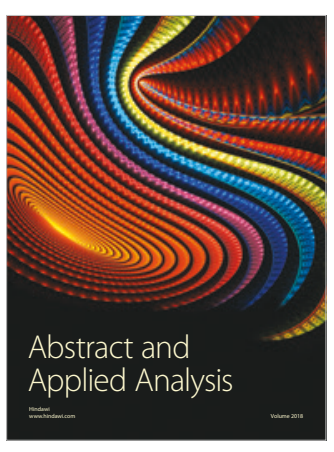

The Scientific

World Journal

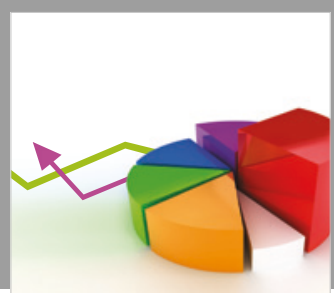

Journal of

Probability and Statistics
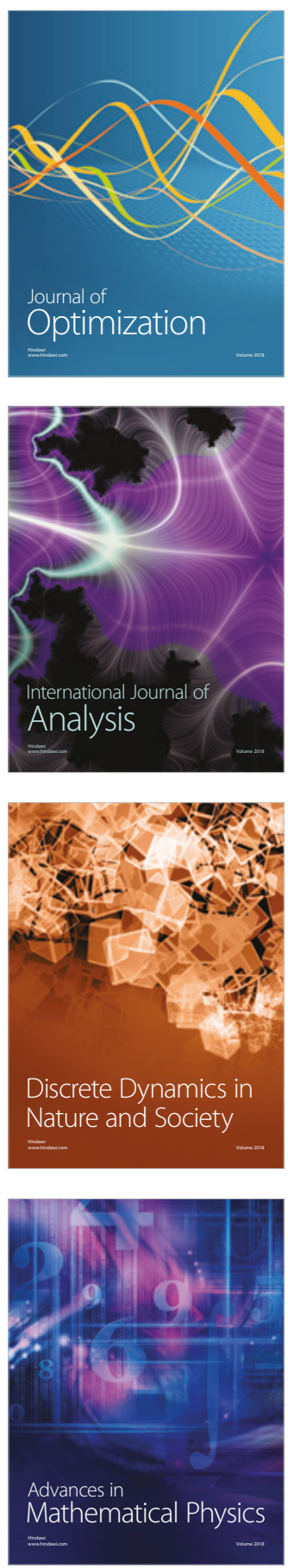